\title{
SUBMANIFOLDS AND THE LENGTH OF THE SECOND FUNDAMENTAL TENSOR
}

\author{
THOMAS HASANIS \\ (Received 4 February 1981; revised 6 July 1981) \\ Communicated by K. Mackenzie
}

\begin{abstract}
A sufficient condition, for a complete submanifold of a Riemannian manifold of positive constant curvature to be umbilical, is given. The condition will be given by an inequality which is established between the length of the second fundamental tensor and the mean curvature.
\end{abstract}

1980 Mathematics subject classification (Amer. Math. Soc.): 53 C 40.

Keywords and phrases: second fundamental tensor, totally umbilical submanifolds.

M. Okumura in [7] gives a sufficient condition for a compact submanifold of a space form to be totally umbilical. This condition is given by an inequality which is established between the length of the second fundamental tensor and the mean curvature. Here we shall prove analogous results (Theorems 1 and 2 below) for non-compact, complete submanifolds of a space form with positive constant curvature. We begin with some auxiliary formulae and known results.

Let $M$ be an $n$-dimensional submanifold of an $(n+p)$-dimensional Riemannian manifold $\bar{M}$ of constant curvature $c$. Let $\nabla$ (respectively $\bar{\nabla}$ ) denote the covariant differentiation in $M$ (respectively $\bar{M}$ ) and let $D$ denote the covariant differentiation in the normal bundle. We will refer to $\nabla$ as the tangential connection and to $D$ as the normal connection. To each unit vector $\xi$ normal to $M$ at a point $q \in M$ is associated a linear transformation of $T_{q} M$ in the following way. Extend $\xi$ to a vector field defined in a neighborhood of $q$ and define $A_{\xi} X$ to be the tangential component of $\bar{\nabla}_{X} \xi$ for $X \in T_{q} M . A_{\xi} X$ depends only on $X$ and $\xi$. Given an orthonormal basis $e_{1}, \ldots, e_{p}$ in the normal bundle, we write $A_{\alpha}=A_{e_{\alpha}}$

Copyright Australian Mathematical Society 1983 
and we call the $A_{\alpha}$ 's the second fundamental tensor associated with $e_{1}, \ldots, e_{p}$. The mean curvature vector $\bar{H}$ of $M$ is defined by

$$
\vec{H}=\frac{1}{n} \sum_{\alpha=1}\left(\operatorname{trace} A_{\alpha}\right) e_{\alpha}
$$

and is independent of our choice of orthonormal basis. We denote the norm of $\vec{H}$ by $H$. We also denote by $S$ the square of the second fundamental tensor of $M$ and we have

$$
S=\sum_{\alpha=1} \operatorname{trace} A_{\alpha}^{2}
$$

which also is independent of our choice of orthonormal basis.

The submanifold $M$ is called totally geodesic if and only if $S=0$ everywhere on $M$. Also if the mean curvature vector $\vec{H}$ is zero everywhere on $M$, then $M$ is called minimal.

Now, for a unit normal $\xi$ we have trace $A_{\xi}^{2} \geqslant \frac{1}{n}\left(\text { trace } A_{\xi}\right)^{2}$. If equality holds then $M$ is called umbilical with respect to $\xi$ and in that case we have $A_{\xi}=$ constant $I$, where $I$ is the identity transformation. If at every point $q \in M$, the submanifold $M$ is umbilical with respect to every normal direction then $M$ is called totally umbilical. It is well known that $M$ is totally umbilical if and only if $S=n H^{2}$ everywhere. It is obvious that a totally geodesic submanifold is totally umbilical.

If $R^{N}$ is the curvature tensor for $D$ then it is well known that

$$
R^{N}(X, Y) e_{\alpha}=\sum_{\beta=1} g\left(\left[A_{\alpha}, A_{\beta}\right] X, Y\right) e_{\beta}
$$

where $X, Y$ are tangents to $M$ and $g$ is the Riemannian metric of $\bar{M}$. We note that $R^{N}=0$ everywhere on $M$ if and only if $\left[A_{\alpha}, A_{\beta}\right]=A_{\alpha} A_{\beta}-A_{\beta} A_{\alpha}=0$ for all $\alpha$, $\beta$; equivalently, the $A_{\alpha}$ 's are simultaneously diagonalizable. If $R^{N}=0$ everywhere we will say that the normal connection $D$ is trivial. A normal vector field $\xi$ is called parallel in the normal bundle if $D \xi=0$; it is obvious that a parallel vector field has constant length.

Finally, let $\nabla^{*}$ denote the sum of the tangential and the normal connections. $\nabla^{*}$ is the connection in the Whitney sum of the tangent and normal bundles induced by $\nabla$ and $D$ ([6], volume I, page 82 ).

The proofs of the results in this paper will consist in simple applications of Omori's Theorem $A^{\prime}$ [8] (see modified form in [4], Theorem B) and of a useful result due to J. Erbacher [3], which we now formulate: Let $M$ be an $n$-dimensional submanifold of an $(n+p)$-dimensional Riemannian manifold $\bar{M}$ of constant 
curvature $c$. If the mean curvature vector $\vec{H}$ is parallel in the normal bundle, then:

$$
\begin{aligned}
\frac{1}{2} \Delta S= & c n S-c \sum_{\alpha=1}\left(\operatorname{trace} A_{\alpha}\right)^{2}+\sum_{\alpha, \beta=1} \operatorname{trace}\left[A_{\alpha}, A_{\beta}\right]^{2} \\
& +\sum_{\alpha, \beta=1}\left(\operatorname{trace} A_{\alpha}\right)\left(\operatorname{trace} A_{\alpha} A_{\beta}^{2}\right) \\
& -\sum_{\alpha, \beta=1}\left(\operatorname{trace} A_{\alpha} A_{\beta}\right)^{2}+\sum_{\alpha=1}\left\|\nabla^{*} A_{\alpha}\right\|^{2}
\end{aligned}
$$

where $\Delta$ is the Laplacian of $M$.

Now, we come to the main results of this paper.

At first we state a lemma which is an easy consequence of a result by Chen-Okumura ([1], page 55 or [2]).

LEMmA. Let $M$ be an $n$-dimensional submanifold of an $(n+p)$-dimensional Riemannian manifold $\bar{M}$ of constant curvature $c$. If the mean curvature vector has constant norm and the norm of the second fundamental tensor is bounded then $M$ has sectional curvatures bounded below.

THEOREM 1. Let $M$ be a complete, connected $n$-dimensional minimal submanifold of an $(n+p)$-dimensional Riemannian manifold $\bar{M}$ (not necessarily complete) of constant positive curvature $c$. If the normal connection of $M$ is trivial then $\sup S \geqslant c n$ or $M$ is a totally geodesic submanifold of $\bar{M}$. In the last case $M$ is compact.

Proof. Let $e_{1}, \ldots, e_{p}$ be an orthonormal basis in the normal bundle. Since the mean curvature vector is zero we have trace $A_{\alpha}=0(\alpha=1, \ldots, p)$. Also, from the triviality of the normal connection we get $\left[A_{\alpha}, A_{\beta}\right]=0$ for all $\alpha, \beta$. Thus from (1) we have:

$$
\frac{1}{2} \Delta S=c n S-\sum_{\alpha, \beta=1}\left(\operatorname{trace} A_{\alpha} A_{\beta}\right)^{2}+\sum_{\alpha=1}\left\|\nabla^{*} A_{\alpha}\right\|^{2}
$$

Now, by using the Cauchy-Schwarz inequality we obtain

$$
\sum_{\alpha, \beta}\left(\operatorname{trace} A_{\alpha} A_{\beta}\right)^{2} \leqslant \sum_{\alpha, \beta}\left(\operatorname{trace} A_{\alpha}^{2}\right)\left(\operatorname{trace} A_{\beta}^{2}\right)=S^{2}
$$

or

$$
-\sum_{\alpha, \beta=1}\left(\operatorname{trace} A_{\alpha} A_{\beta}\right)^{2} \geqslant-S^{2}
$$


and thus (2) becomes

$$
\frac{1}{2} \Delta S \geqslant c n S-S^{2}+\sum_{\alpha=1}\left\|\nabla^{*} A_{\alpha}\right\|^{2}
$$

or

$$
\frac{1}{2} \Delta S \geqslant S(c n-S) .
$$

It is sufficient to prove the following: If $\sup S<c n$, then $M$ must be totally geodesic. In fact, if $\sup S<c n$, then there exists a positive number $\varepsilon$ such that $S \leqslant c n-\varepsilon$, everywhere on $M$ and thus the sectional curvatures of $M$ are bounded below (see the lemma). Also $c n-S \geqslant \varepsilon$ and (3) gives

$$
\frac{1}{2} \Delta S \geqslant S \varepsilon .
$$

Since $S$ is bounded above and the sectional curvatures of $M$ are bounded below, we claim that $S=0$, everywhere on $M$. In fact, if for some point $q_{1}$ we had $S\left(q_{1}\right)=b>0$, then from (4) we would have $\frac{1}{2} \Delta S(q) \geqslant b \varepsilon=$ const $>0$ for all points $q \in M$ such that $S(q) \geqslant S\left(q_{1}\right)$, which contradicts Omori's theorem. Thus $S=0$ and so $M$ is totally geodesic. Moreover $M$ is totally umbilical and by a well known result (see [1], page 49) $M$ has constant curvature $c$. Since $M$ is complete it must be compact.

Remark 1. An analogous result to Theorem 1 has been proved in [5], without the assumption of the triviality of the normal bundle. But in that case the bound for $\sup S$ is weaker.

THEOREM 2. Let $M$ be an $n$-dimensional $(n \geqslant 3)$ complete, connected submanifold of an $(n+p)$-dimensional Riemannian manifold $\bar{M}$ (not necessarily complete) of positive constant curvature $c$, whose mean curvature vector is non-zero and parallel with respect to the induced connection of the normal bundle. If the normal connection is trivial and the second fundamental tensor $A_{\alpha}(\alpha=1, \ldots, p)$ satisfies

$$
\sum_{\alpha=1} \operatorname{trace} A_{\alpha}^{2} \leqslant \frac{1}{n-1} \sum_{\alpha=1}\left(\operatorname{trace} A_{\alpha}\right)^{2}+2 c \quad\left(\text { that is } S \leqslant \frac{n^{2} H^{2}}{n-1}+2 c\right),
$$

then $M$ is totally umbilical. Moreover $M$ is compact.

Proof. We choose an orthonormal basis $e_{1}, \ldots, e_{p}$ in the normal bundle such that $e_{1}$ is the unit vector in the direction of $\vec{H}$. Then trace $A_{1}=n H$ and trace $A_{\alpha}=0$ for $\alpha=2, \ldots, p$. Also by using Theorem 4 in [5] we conclude that $A_{1}=H I$ (since $M$ is umbilical with respect to $e_{1}$ ) and thus trace $A_{1}^{2}=n H^{2}$ and 
trace $A_{1}^{3}=n H^{3}$. Moreover by using the triviality condition we get from (1) that:

$$
\begin{aligned}
\frac{1}{2} \Delta S= & c n S-c n^{2} H^{2}+n H \sum_{\beta=1}\left(\operatorname{trace} A_{1} A_{\beta}^{2}\right) \\
& -\sum_{\alpha, \beta=1}\left(\operatorname{trace} A_{\alpha} A_{\beta}\right)^{2}+\sum_{\alpha=1}\left\|\nabla^{*} A_{\alpha}\right\|^{2}
\end{aligned}
$$

or

$$
\begin{aligned}
\frac{1}{2} \Delta S= & c n S-c n^{2} H^{2}+n H^{2} \sum_{\beta=2}\left(\operatorname{trace} A_{\beta}^{2}\right) \\
& -\sum_{\alpha, \beta=2}\left(\operatorname{trace} A_{\alpha} A_{\beta}\right)^{2}+\sum_{\alpha=1}\left\|\nabla^{*} A_{\alpha}\right\|^{2} .
\end{aligned}
$$

We set $\Sigma_{\beta=2}$ trace $A_{\beta}^{2}=f$. Then $S=n H^{2}+f$ and by the constancy of $H$ we get from (6):

$$
\frac{1}{2} \Delta f=c n f+n H^{2} f-\sum_{\alpha, \beta=2}\left(\operatorname{trace} A_{\alpha} A_{\beta}\right)^{2}+\sum_{\alpha=1}\left\|\nabla^{*} A_{\alpha}\right\|^{2}
$$

or since

$$
\sum_{\alpha, \beta=2}\left(\operatorname{trace} A_{\alpha} A_{\beta}\right)^{2} \leqslant \sum_{\alpha, \beta=2}\left(\operatorname{trace} A_{\alpha}^{2}\right)\left(\operatorname{trace} A_{\beta}^{2}\right)=f^{2}
$$

we get

(7) $\frac{1}{2} \Delta f \geqslant f\left(c n+n H^{2}-f\right)=f\left\{\frac{n H^{2}}{n-1}+2 c+(n-2)\left[c+\frac{n H^{2}}{n-1}\right]-f\right\}$.

Now, from (5) and trace $A_{1}^{2}=n H^{2}$ we have $n H^{2} /(n-1)+2 c-f \geqslant 0$ and thus from (7) we obtain

$$
\frac{1}{2} \Delta f \geqslant f \varepsilon_{0}, \quad \text { where } \varepsilon_{0}=(n-2)\left[c+\frac{n H^{2}}{n-1}\right] \text { is a positive constant. }
$$

We proceed as in the proof of Theorem 1 and we conclude that $f=0$, or trace $A_{\alpha}^{2}=0$ for all $\alpha=2, \ldots, p$. Thus $S=n H^{2}$, namely $M$ is totally umbilical. Moreover, as in Theorem 1 we conclude that $M$ has constant curvature $c+H^{2}$ and thus it is compact.

Remark 2. When $p=2$, the triviality condition in Theorem 2 is satisfied automatically ([3], Lemma 7 in page 27).

REMARK 3. Theorem 2 obviously generalizes the results of [7] in some directions. 


\section{References}

[1] B.-Y. Chen, Geometry of submanifolds (Marcel Dekker, New York, 1973).

[2] B.-Y. Chen and M. Okumura, 'Scalar curvature, inequality and submanifold', Proc. Amer. Math. Soc. 38 (1973), 605-608.

[3] J. Erbacher, Isometric immersions of Riemannian manifolds into space forms (Thesis, Brown University, 1970).

[4] Th. Hasanis, 'Isometric immersions into spheres', J. Math. Soc. Japan 33 (1981), 551-555.

[5] Th. Hasanis, 'Submanifolds and a pinching problem on the second fundamental tensors', to appear.

[6] S. Kobayashi and K. Nomizu, Foundations of differential geometry, Vols. I, II (John Wiley and Sons, Inc., 1963, 1969).

[7] M. Okumura, 'Submanifolds and a pinching problem on the second fundamental tensors', Trans. Amer. Math. Soc. 178 (1973), 285-291.

[8] H. Omori, 'Isometric immersions of Riemannian manifolds', J. Math. Soc. Japan 19 (1967), 409-415.

\section{Department of Mathematics}

University of Ioannina

Ioannina

Greece 